\section{Orofacial presentations of sarcoidosis - a case series and review of the literature}

\author{
T. W. J. Poate, ${ }^{1}$ R. Sharma, ${ }^{2}$ K. A. Moutasim, ${ }^{3}$ M. P. Escudier ${ }^{4}$ \\ and S. Warnakulasuriya ${ }^{5}$
}

VERIFIABLE CPD PAPER
IN BRIEF
- Demonstrates the wide spectrum of orofacial manifestations of sarcoidosis above and beyond the 'classical' features of the disease.
- Serves as an aide memoire for junior hospital clinicians, general dental practitioners and dental students.
- Highlights the dental aspects of this systemic condition and is beneficial when formulating treatment plans for patients affected by the disorder.

Sarcoidosis is a multi-system disease of unknown aetiology characterised by the presence of non-caseating granulomas, the lungs and lymph nodes being the most affected sites. Orofacial manifestations of the condition are increasingly recognised, with several recent case reports where the initial presentation of the disease is in the region. Here, we report six cases of orofacial sarcoidosis which help to illustrate the wide spectrum of the condition.

\section{INTRODUCTION}

Sarcoidosis is a systemic granulomatous condition of unknown cause affecting multiple organs. ${ }^{1}$ The disease is characterised by the presence of non-caseating granulomas and whilst any organ may be affected the most common sites are the lungs and lymph nodes. ${ }^{2}$ More than 3,000 new cases of sarcoidosis are diagnosed in the UK each year with a higher incidence among young adults aged 20-40..$^{2,3}$ It occurs in all races but appears to be more common in people of African-American, Afro-Caribbean or Scandinavian origin. ${ }^{2,4,5}$

It has been suggested that sarcoidosis is due to an immunological response to an infective trigger in a genetically predisposed individual, with the most likely candidates being mycobacteria and propionibacteria. ${ }^{5}$

Affected patients may be asymptomatic or have a wide range of nonspecific symptoms depending on the organs affected and stage of the disease.

\footnotetext{
${ }^{1}$ Consultant in Oral Medicine; ${ }^{2}$ Senior House Officer in Oral Medicine; ${ }^{3 *}$ Speciality Dentist in Oral Medicine, Barts \& The London NHS Trust, London; ${ }^{4}$ Consultant in Oral Medicine; ${ }^{5}$ Professor of Oral Medicine, Dental Institute at Guy's, King's and St. Thomas' Hospitals, London

${ }^{*}$ Correspondence to: Dr Karwan Moutasim Email:k.moutasim@qmul.ac.uk
}

\section{Refereed Paper}

Accepted 11 July 2008

DOI: $10.1038 /$ sj.bdj.2008.892

${ }^{\circledR}$ British Dental Journal 2008; 205: 437-442
The lungs are often affected and in such cases the patient may present with a dry cough, dyspnoea or chest pain. Other common symptoms include a flu-like illness with fever, night sweats, fatigue and arthralgia. Ocular discomfort and reduced visual acuity due to uveitis may occur as may lymphadenopathy and enlargement of the liver and spleen. Cutaneous lesions may also be present and include a macular papular rash and erythema nodosum. ${ }^{2}$

Oral involvement has been noted as the initial manifestation in several case reports of sarcoidosis. ${ }^{6-8}$

We report six cases of orofacial sarcoidosis that have attended the oral medicine department in this dental institute in London in the past 24 months which help to illustrate the spectrum of the orofacial presentations of this condition.

\section{CASE 1: TRIGEMINAL NEUROPATHY}

A 33-year-old Caucasian lady was referred to the oral medicine department by her general dental practitioner with a one year history of numbness of the left side of the face and forehead. She had also noticed an asymptomatic lump on the left side of her tongue which had been present for the last three months. She had also recently been diagnosed with hypertrophic cardiomyopathy but had no other relevant medical history. She had no chest symptoms.

On examination she was found to have reduced sensation to light touch and pin prick in the distribution of all three branches of the left trigeminal nerve. The left corneal reflex was also reduced. The motor component of the nerve was spared and neurological examination was otherwise normal. There was a $6 \times 7$ $\mathrm{mm}$ firm swelling on the left lateral border of the tongue. An excisional biopsy of the lingual swelling demonstrated the presence of non-caseating granulomas suggestive of sarcoidosis (Fig. 1). Her serum angiotensin converting enzyme (SACE) was raised at $110 \mathrm{U} / \mathrm{L}$ (normal level 27-82 U/L).

A diagnosis of sarcoidosis was made and she was referred for chest and neurology opinions. Clinical examination of the chest was unremarkable whilst a chest X-ray revealed slight hilar prominence. Tuberculosis was excluded by the mantoux test. An MRI scan demonstrated a mass adjacent to the left trigeminal ganglion. Treatment was commenced with prednisolone at a dose of $40 \mathrm{mg}$ with appropriate prophylaxis including a bisphosphonate.

At her subsequent review by a cardiologist it was felt that her cardiac disease was probably a further manifestation of the sarcoidosis. Over the next four months her facial sensation improved. A repeat MRI showed the mass in the trigeminal ganglion to have reduced in size and her prednisolone was therefore gradually reduced and then stopped over the following six months without relapse. 


\section{CASE 2: SALIVARY AND LACRIMAL GLAND SWELLING}

A 43-year-old Indian female developed bilateral parotid and submandibular swelling. This was initially diagnosed by her general medical practitioner (GMP) as mumps and was managed conservatively. She subsequently developed a right sided lower motor neurone lesion of the seventh cranial nerve for which she was prescribed a two week course of prednisolone with good effect. However, three weeks after the cessation of the prednisolone the bilateral parotid and submandibular swelling recurred. On this occasion she was also noted to have bilateral lacrimal swelling. In view of this the GMP recommenced prednisolone and referred her to a local physician. Further investigations including liver function tests and SACE were within normal limits as were a chest $\mathrm{X}$-ray and CT scan. In view of the persisting salivary gland swelling she was referred to the oral medicine department for further investigation.

On further questioning she reported dryness of the mouth and eyes. She also had arthralgia, weight loss and blurred vision which had worsened recently. She had no chest symptoms. On examination she had no swelling of the parotid, submandibular or lacrimal glands. She had slightly reduced visual acuity in the right eye and was noted to have 'shotty' cervical lymphadenopathy. Intra-orally she had a well lubricated and generally healthy oral mucosa. There was a good flow of clear saliva from all major salivary gland duct orifices. A provisional diagnosis of sarcoidosis (Heerfordt's syndrome) was made based on the history of salivary gland swelling, lacrimal involvement and seventh nerve palsy whilst the differential diagnosis included Sjögren's syndrome.

The unstimulated whole salivary flow and stimulated parotid salivary flow rates were within normal limits. Schirmer's test was negative for both eyes. Her autoimmune profile including Ro and La antoantibodies was negative. The SACE was repeated and on this occasion found to be raised at $94 \mathrm{U} / \mathrm{L}$ (normal level 27-82 U/L). Ultrasound examination demonstrated an enlarged jugulo-digastric lumph node and multiple hypoechoic areas within the parotid

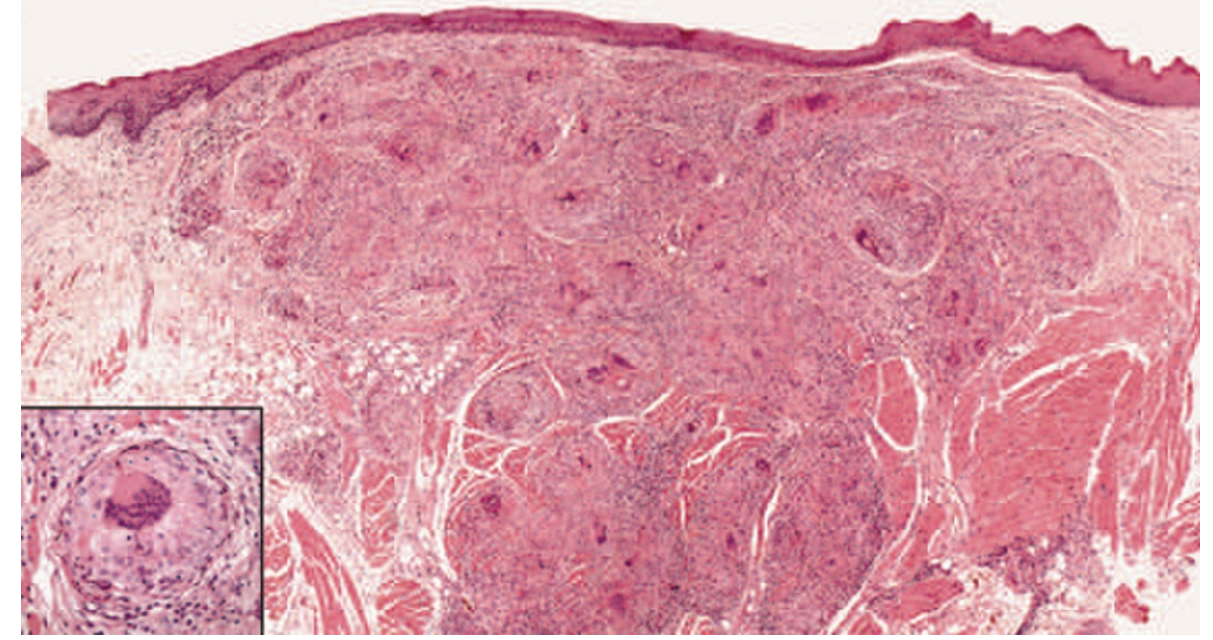

Fig. 1 High-power photomicrograph of lingual mucosa demonstrating non-caseating granulomas of sarcoidosis (case 1). An individual granuloma is shown in the left corner (haematoxylin \&t eosin)

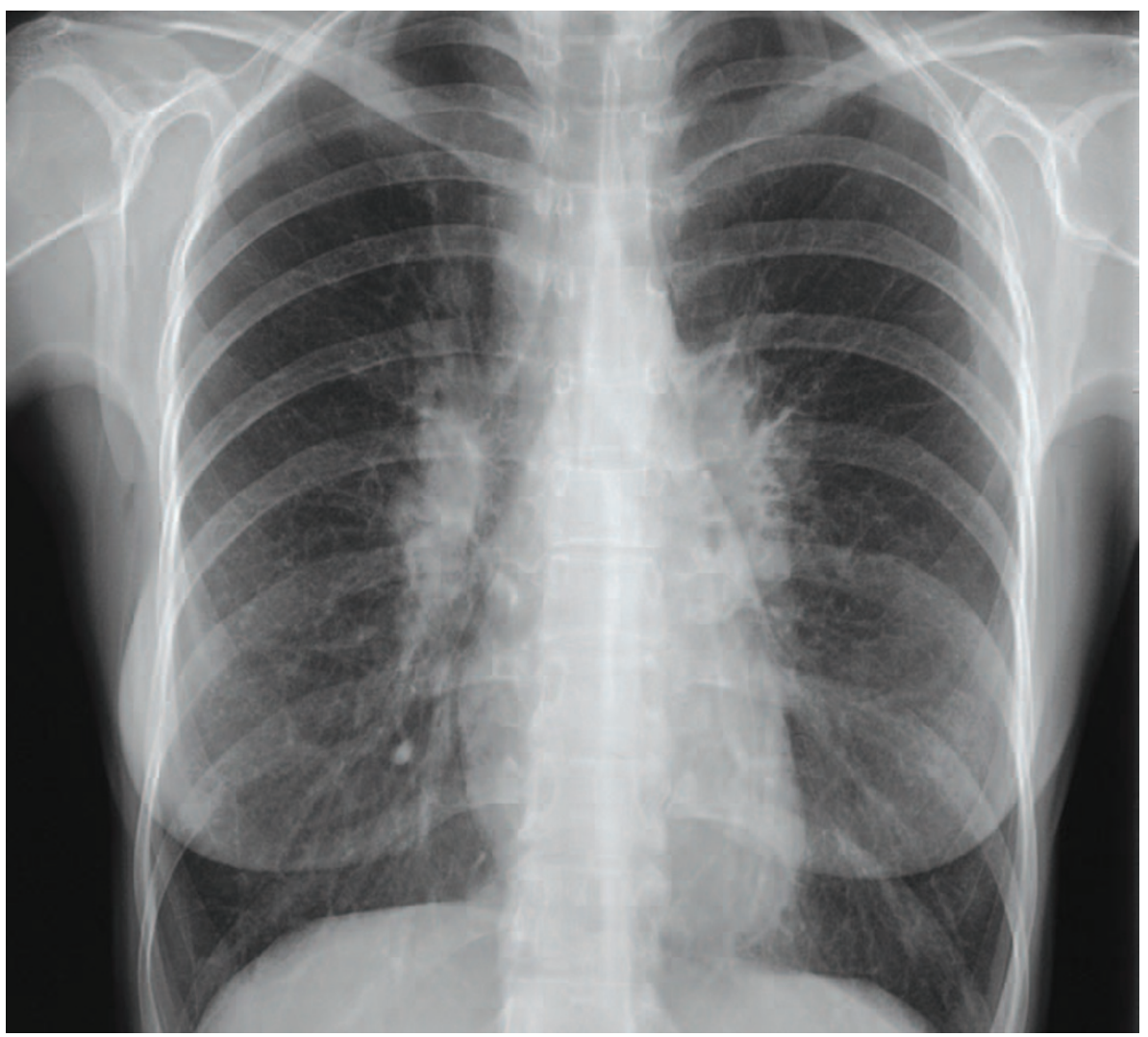

Fig. 2 Anteroposterior chest radiograph showing bilateral hilar lymphadenopathy with borderline nodularity in case 2

and submandibular glands which were highly vascular. Parotid sialography was essentially normal with no delay of emptying or sialectasis. A chest x-ray showed bilateral hilar lymphadenopathy with borderline nodularity (Fig. 2) and she was subsequently referred to a chest physician and ophthalmologist. Formal pulmonary function tests were normal whilst a fluorescein angiogram showed optic disc hyperfluorescence and some leakage indicating activity in the optic nerve head. The patient declined any further investigations and a diagnosis of salivary and ocular sarcoidosis was made. In view of her persisting symptoms oral prednisolone was continued for two months before being reduced and then stopped as her symptoms resolved. She has since remained symptom free for two years with no further active treatment required. 


\section{CASE 3: PAROTID GLAND}

\section{SWELLING}

A 27-year-old gentleman of mixed ethnicity with a Caucasian mother and African father was admitted under the care of the neurologists with a three month history of generalised muscle aching and weakness in his legs causing difficulty in walking up stairs. He also had bilateral swelling of the parotid glands for which an oral medicine opinion was requested. He had previously been prescribed non-steroidal anti-inflammatory drugs by his GMP which had improved his symptoms over a period of six weeks. He then developed a flulike illness, fever, productive cough, widespread rash and cervical lymphadenopathy with associated difficulty in swallowing. On this occasion his GMP prescribed a course of antibiotics but his symptoms persisted and he went on to develop night sweats and reported two stone weight loss in eight weeks. He also reported dryness of the mouth.

His medical history included asthma for which he used a salbutamol inhaler and eczema. He had also been diagnosed at the age of seven years with temporal lobe epilepsy which was controlled with carbamazepine and vigabatrin. He was a non-smoker and did not drink alcohol. He had not travelled abroad for several years.

On examination he had a widespread annular erythematous dry scaly rash on his trunk and all four limbs. He was pyrexial and was noted to have marked non-tender bilateral parotid swelling (Fig. 3). He had widespread cervical, axillary and inguinal lymphadenopathy. Respiratory and abdominal examination was unremarkable. Neurological examination revealed increased tone in the left leg with brisk reflexes at the knees and ankles bilaterally. Vibration sense was reduced in the left toes and ankle and pinprick sensation was reduced over the sole of the left foot. His gait was abnormal and he walked with a semi-spastic gait. Cranial nerves were intact.

Blood tests revealed a normocytic anaemia with a haemoglobin of 11.6 $\mathrm{g} / \mathrm{dl}$ (normal range $13.0-16.5 \mathrm{~g} / \mathrm{dl}$ ). Liver function tests were abnormal with gamma-glutamyl transferase at $885 \mathrm{IU} /$ L (1-55 IU/L), aspartate transaminase at $69 \mathrm{IU} / \mathrm{L}$ (10-50 IU/L) and alkaline phosphatase at $497 \mathrm{IU} / \mathrm{L}$ (30-130 IU/L). The C-reactive protein (CRP) was raised at $98 \mathrm{mg} / \mathrm{L}(<5 \mathrm{mg} / \mathrm{L})$. Blood cultures were negative and HIV, hepatitis B and $\mathrm{C}$ and tuberculosis were excluded. Serology for toxoplasma, cytomegalovirus, Epstein Barr virus and brucella was unremarkable and an autoantibody screen was negative. SACE was raised at $224 \mathrm{U} / \mathrm{L}$ (8-52 U/L).

Ultrasound examination demonstrated both parotid glands to be enlarged and inflamed with non-specific features of parotitis. Reactive lymph nodes were noted at the level of the carotid bifurcation bilaterally. MRI demonstrated a normal intracranial appearance with marked enlargement of both parotid glands. Chest X-ray was unremarkable whilst a CT scan demonstrated bilateral, enlarged axillary, peri-portal and inguinal lymphadenopathy. A gallium scan showed increased uptake of tracer in the liver, lacrimal glands, central nasal region, parotid glands and submandibular glands as well as in the axillary and inguinal lymphatic chains.

Incisional biopsy of the parotid gland demonstrated multiple non-caseating epithelioid granulomas surrounding the ducts and destroying the acinar structures. Similar granulomas were present in a skin biopsy. A diagnosis of multi-system sarcoidosis with cutaneous, hepatic, neurological and salivary gland involvement was made. He was reviewed by a respiratory physician and commenced on prednisolone $60 \mathrm{mg}$ daily with bisphosphonate and calcium supplementation. His skin rash improved within a few days and after three months the SACE and CRP had returned to normal while the liver function tests had improved considerably. His prednisolone was reduced at this stage to $20 \mathrm{mg}$ daily. A further three months later his neurological deficits, parotid swelling and oral dryness are improving and continue to be monitored.

\section{CASE 4: XEROSTOMIA}

A 35-year-old Afro-Caribbean lady was referred to the oral medicine department with a two year history of a dry mouth and gritty eyes. She was taking frequent sips of water and was using artificial tears daily. She denied any facial

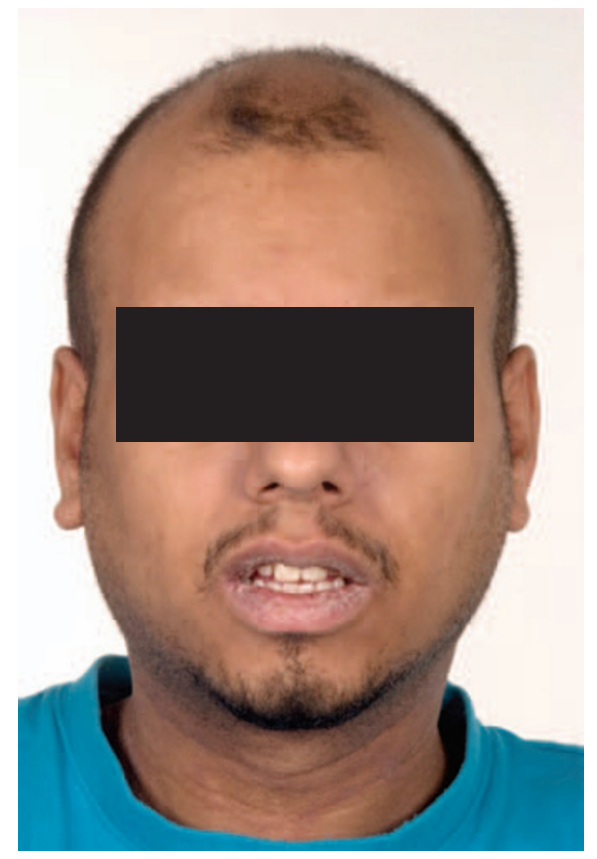

Fig. 3 Bilateral parotid swelling in case 3

swelling, cutaneous or genital dryness or joint symptoms. She also had fatigue and a persistent, unproductive cough for which she had been referred to the chest department. She was a non-smoker. She was known to have macular degeneration and sickle cell trait and to be iron deficient for which she was taking iron supplements.

On examination she had no facial swelling but had dryness of the oral mucosa with lack of saliva pooling and reduced quantities of saliva milkable from the major salivary ducts. The unstimulated whole salivary flow rate was $0.1 \mathrm{ml} /$ minute (normal value $>0.2$ $\mathrm{ml} /$ minute). She was negative for Ro and La autoantibodies. The SACE was raised at $225 \mathrm{U} / \mathrm{L}$ (normal level 27-82 U/ L). There was also derangement of liver enzymes with alkaline phosphatase 276 IU/L (30-130 IU/L), aspartate transaminase 58 IU/L (10-50 IU/L), GGT 151 IU/L (1-55 IU/L). The white cell count was low at $3.89 \times 10^{9}$ (normal value 4.0 - $11.0 \times 10^{9}$ ) with a neutropenia and lymphopenia. Ultrasound of the parotid glands demonstrated small hypoechoic areas throughout the parenchyma but sialography showed no abnormality in either parotid gland and did not support a diagnosis of Sjögren's syndrome. A labial gland biopsy reported the presence of non-caseating granulomas. A chest X-ray was reported as showing bilateral hilar lymphadenopathy and a 
reticular nodular pattern of increased lung markings seen throughout both lungs consistent with pulmonary parenchymal sarcoidosis. Pulmonary function tests demonstrated a restrictive pulmonary defect. CT scan demonstrated paratracheal lymphadenopathy. She was referred to the liver unit where a liver biopsy was performed which confirmed a granulomatous hepatitis. Ocular assessment excluded ocular sarcoid.

A diagnosis of multi system sarcoidosis with pulmonary, bone marrow, hepatic and salivary gland involvement was made. She was advised to use sugar free gum and prescribed saliva stimulating tablets for the symptomatic control of her dry mouth. She was also commenced on prednisolone $30 \mathrm{mg}$ daily by the chest physicians. This led to a progressive improvement in her chest symptoms and a return of the SACE level to within normal limits. One year later she remains on prednisolone at a reduced dose of 15 $\mathrm{mg}$ daily, has no chest symptoms with a normal chest X-ray and improved pulmonary function tests.

\section{CASE 5: GINGIVAL SWELLING}

A 41-year-old Afro-Caribbean lady was referred to the oral medicine department by the department of oral surgery following the extraction of her wisdom teeth. At the time of surgery it was noted that she had generalised swelling of her gingivae despite good oral hygiene although the patient had been unaware of this. She suffered from sickle cell trait and glucose-6-phosphate deficiency but was otherwise fit and well with no chest symptoms. On examination she had generalised swelling of the gingivae with areas of erythema (Fig. 4). An incisional biopsy was performed which reported that the corium was almost completely replaced by numerous, non-caseating granulomas consistent with a diagnosis of sarcoidosis. She was referred to a chest physician who found no evidence of chest involvement. In view of the absence of any symptoms or extra-oral involvement no treatment was commenced.

Five years later she developed shortness of breath and a chest X-ray showed her to have bilateral hilar lymphadenopathy. In view of this she was diagnosed

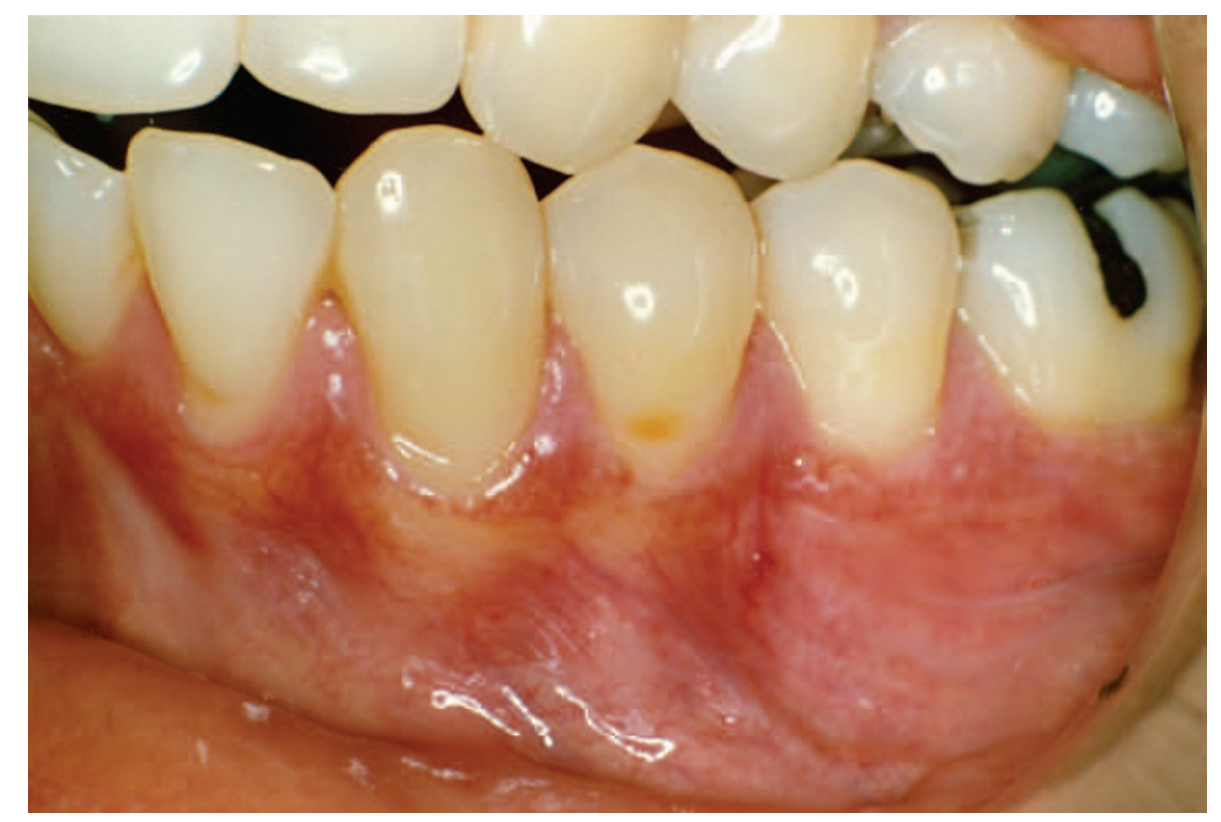

Fig. 4 Intraoral photograph showing gingival swelling with mild erythema in sarcoidosis (case 5)

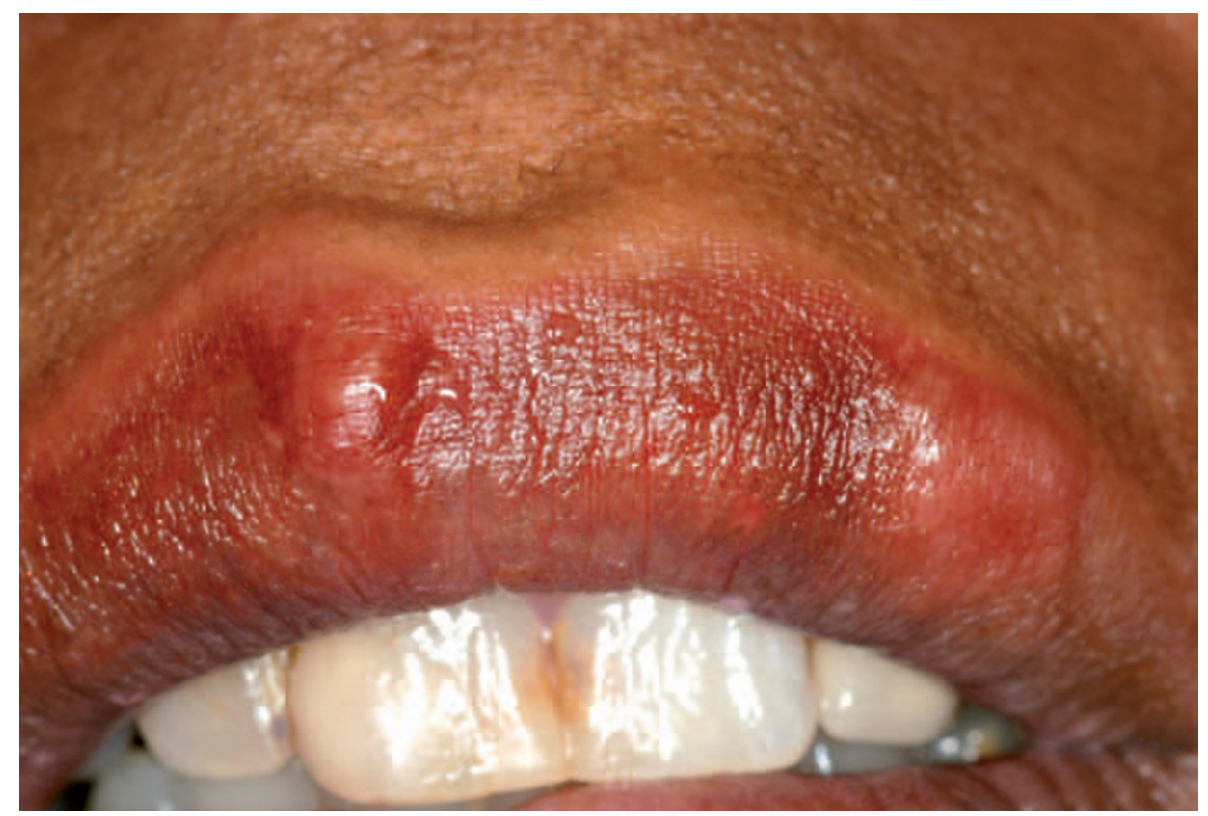

Fig. 5 Two distinct sarcoid nodules affecting the upper lip in case 6

as having pulmonary sarcoidosis and commenced on prednisolone with good effect. At the present time she remains on therapy and under review.

\section{CASE 6: LIP AND PALATAL SWELLING}

A 43-year-old African lady was referred to the oral medicine department with a recurrence of a swelling on the upper lip. This was causing her considerable social embarrassment and occasional discomfort when eating. She had had a nodule surgically excised from the same site one year earlier which had been confirmed as sarcoidosis. A further upper lip nodule had been treated with intralesional steroid injections and had reduced in size. She had previously been diagnosed with cutaneous sarcoidosis affecting the legs and face, whilst biopsies of the lower lip at that time had also been reported as sarcoid deposits. Further similar deposits had also been removed from above the anterior commissure in the supraglottis. Recent lung function tests were within normal limits although CT scan had shown extensive mediastinal lymphadenopathy but little in the way of parenchymal changes. She had at no stage been given systemic therapy for the sarcoidosis.

On examination she had a scar on the upper lip at the site of the previous exci- 
sion. There were two distinct nodules affecting the upper lip, swelling of the midline of the lower lip and a further swelling of the soft palate (Fig. 5). Due to the position of the upper lip swellings and the patient's wishes to avoid further scarring they were treated with intralesional triamcinolone, resulting in a reduction of their size. No treatment was considered necessary for the swellings of the lower lip or soft palate. At the present time we are awaiting her review six months after this procedure to assess if repeat injection or further intervention is required.

\section{DISCUSSION}

Oral involvement in sarcoidosis is rare. A recent review of the English literature found only 68 well-documented cases of oral sarcoidosis. ${ }^{9}$ Of these 39 cases were female and 25 were male with the data being incomplete in the other four cases. The jaws were affected in 21 cases (maxilla nine, mandible eight, maxilla and mandible four). Presenting features in these cases were loosening of the teeth, pain, nasal obstruction, swelling of the mandible, maxillary bone loss and non-healing socket. The soft tissues of the oral cavity were affected in 47 cases, with the buccal mucosa being the most commonly affected soft tissue site. The common clinical presentations were localised swellings or nodules, ulcers, gingivitis, gingival hyperplasia and gingival recession. Soft tissue involvement of the oral cavity presented as a firm swelling affecting the lips, gingivae, floor of mouth, tongue, hard or soft palate, buccal mucosa and vestibule.

Salivary gland involvement has also been reported with sarcoidosis presenting as swelling of the parotid glands, ${ }^{10,11}$ submandibularglands ${ }^{11}$ and xerostomia. ${ }^{12}$ A recent case report describes a case of sarcoidosis presenting with xerostomia, dysgeusia, oral burning, xerophthalmia and bilateral swelling. ${ }^{7}$ Heerfordt's syndrome is a form of systemic sarcoidosis characterised by swelling of the parotid glands, uveitis and facial nerve palsy. ${ }^{13}$ Involvement of the minor salivary glands in patients with sarcoidosis has been reported to range from $19-58 \% 0^{14}$ and may help differentiate sarcoidosis from Sjögren's syndrome. ${ }^{15}$
Oral involvement may be the initial manifestation of the sarcoidosis. ${ }^{6-8}$ In one of the cases described in this series the diagnosis of oral sarcoidosis preceded the need for systemic treatment for pulmonary involvement by five years.

The diagnosis of sarcoidosis is established by the presence of clinical features combined with the supporting histopathological finding of non caseating granulomas. ${ }^{1}$ Serum ACE may be raised in active sarcoidosis as this enzyme is produced by granulomas. However the level of this enzyme is only raised in 50-80\% of patients with sarcoidosis. ${ }^{16,17}$ In the second case presented in this paper the serum ACE was initially within normal limits but later became raised with the progression of the condition. The measurement of this enzyme may be useful in monitoring the response to treatment. A biopsy of affected tissue should be performed as the demonstration of noncaseating granulomas strongly supports the diagnosis of sarcoidosis, although other granulomatous conditions such as tuberculosis, orofacial granulomatosis, foreign body reactions and deep seated fungal infections need to be excluded.

In cases of orofacial sarcoidosis referral for chest and ophthalmological assessment is important. A chest X-ray should also be performed to investigate for the presence of hilar lymphadenopathy. The involvement of other specialities may be indicated by clinical features and investigation results. Other imaging techniques may be useful in the diagnosis of sarcoidosis. CT may demonstrate the presence of mediastinal and hilar lymphadenopathy and pulmonary or neurological involvement. Magnetic resonance imaging (MRI) may show organ involvement including neurological, muscle and bone disease. Contrast enhancement with gadolinium improves the sensitivity of the technique. Positron emission tomography (PET) scanning with markers has also been used., ${ }^{1,18}$

Treatment of sarcoidosis may range from observation in asymptomatic or mild disease to systemic corticosteroid therapy in more severe cases. Systemic therapy is particularly needed for patients with neurological or ocular involvement or progressive respiratory disease. Steroid sparing agents used for sarcoidosis include chloroquine and hydroxychloroquine, ${ }^{19}$ methotrexate, ${ }^{20}$ azathioprine, ${ }^{21}$ pentoxyfylline, ${ }^{22}$ and thalidomide. ${ }^{23}$ Infliximab, a monoclonal antibody targeted against TNF $\alpha$ has been found to be helpful in refractory sarcoidosis. ${ }^{24,25}$

Orofacial sarcoidosis may require no treatment but cases requiring treatment frequently respond to corticosteroid therapy which may be administered intralesionally or systemically. In some cases surgical excision of granulomatous swellings may be required..$^{26} \mathrm{Sim}$ ple symptomatic treatment to stimulate or replace saliva may help in cases of xerostomia.

Orofacial presentations of sarcoidosis are rare. It is, however, important that the wide range of presentations of sarcoidosis in the orofacial region are recognised as they may indicate the presence or future development of systemic involvement and the need for systemic therapy.

The authors would like to thank Professor S. J. Challacombe and Dr P. J. Shirlaw for permission to present their cases.

1. Baughman R, Lower E E, Du Bois R M. Sarcoidosis. Lancet 2003; 61: 1111-1118.

2. Thomas K W, Hinninghake G W. Sarcoidosis. JAMA 2003; 289: 3300-3303.

3. Gribbin J, Hubbard R, Le Jeune I, Smith C J P et al. Incidence and mortality of idiopathic pulmonary fibrosis and sarcoidosis in the UK. Thorax Epub 2006 July 14.

4. Rybicki B A, Major M, Popovich J Jr, Maliarik M J, lannuzzi M C. Racial differences in sarcoidosis incidence: a 5-year study in a health maintenance organization. Am J Epidemiol 1997; 145: 234-241.

5. Du Bois R M, Goh N, McGrath P, Cullivan P. Is there a role for microorganisms in the pathogenesis of sarcoidosis? J Int Med 2003; 253: 4-17.

6. Blinder D, Yahatom R, Taicher S, Hashomer T. Oral manifestations of sarcoidosis. Oral Surg Oral Med Oral Pathol Oral Radiol Endod 1997; 83: 458-461.

7. Fatahzadeh M, Rinaggio J. Diagnosis of systemic sarcoidosis prompted by orofacial manifestations. J Am Dent Assoc 2006; 137: 54-60.

8. Jackowski J, Dragistic D, Arnold G, Dirschka T. Primary oral sarcoidosis preceding Lofgren's syndrome. Oral Surg Oral Med Oral Pathol Oral Radiol Endod 2005; 100: 183-185.

9. Suresh L, Radfar L. Oral sarcoidosis: a review of literature. Oral Dis 2005; 11: 138-145.

10. Nitzan D W, Shteyer A. Sarcoidosis of the parotid salivary glands. J Oral Maxillofac Surg 1982; 40: 443-446.

11. Vairaktaris E, Vassiliou S, Yapijakis C, Papakosta V et al. Salivary gland manifestations of sarcoidosis: report of three cases. J Oral Maxillofac Surg 2005; 63: 1016-1021.

12. Chisholm D M, Lyell A, Haroon TS, Mason D K, Beeley J A. Salivary gland function in Sarcoidosis. Report of a case. Oral Surg Oral Med Oral Pathol 1971; 31: 766-771

13. Bopp F P, Cheney M L, Donzis P B, White J A, Reed H T. Heerfordt syndrome: a cause of facial paralysis. J La State Med Soc 1990; 142: 13-15.

14. Nessan V J, Jacoway J R. Biopsy of minor salivary glands in the diagnosis of sarcoidosis. N Eng/ J Med 1979; 301: 922-924. 
15. Giotaki H, Constantopoulus S H, Papadimimitriou CS, Moutsopoulos H M. Labial minor salivary gland biopsy: a highly discriminatory diagnostic method between sarcoidosis and Sjögren's syndrome. Respiration 1986; 50: 102-107.

16. Turton C W, Grundy E, Firth G, Rigden B G, Smyth $J$ T, Turner-Warwick M. Value of measuring angiotensin I converting enzyme and serum lysozyme in the management of sarcoidosis. Thorax 1979; 34: 57-62.

17. Khan A H, Ghani F, Khan A, Khan M A, Khurshid M. Role of serum angiotensin converting enzyme in sarcoidosis. J Pak Med Assoc 1998; 48: 131-133.

18. Lebtahi R, Crestani B, Belmatoug N et al. Somato- statin receptor scintigraphy and gallium scintigraphy in patients with sarcoidosis. J Nucl Med 2001; 42: $21-26$.

19. Sharma O P. Effectiveness of chloroquine and hydroxychloroquine in treating selected patients with sarcoidosis with neurologic involvement. Arch Neurol 1998; 55: 1248-1254.

20. Lower E E, Baughman R P. Prolonged use of methotrexate for sarcoidosis. Arch Intern Med 1995; 155: 846-851.

21. Muller-Quernheim J, Kienast K, Held M, Pfeifer S, Costabel U. Treatment of chronic sarcoidosis with an azathioprine/prednisolone regimen. Eur Respir J 1999; 14: 1117-1122.
22. Zabel P, Entzian P, Dalhoff K, Schlaak M. Pentoxifylline in treatment of sarcoidosis. Am J Respir Crit Care Med 1997: 155: 1665-1669.

23. Baughman R P, Judson M A, Teirstein A S, Moller D $\mathrm{R}$, Lower $\mathrm{E} E$. Thalidomide for chronic sarcoidosis. Chest 2002; 122: 227-232.

24. Baughman R P, Lower E E. Infliximab for refractory sarcoidosis. Sarcoidosis Vasc Diffuse Lung Dis 2001; 18: 70-74

25. Doty J D, Mazur J E, Judson M A. Treatment of sarcoidoisis with infliximab. Chest 2005; 127: 1064-1071.

26. Steinberg M J, Mueller D P. Treating oral sarcoidosis. J Am Dent Assoc 1994; 125: 76-79. 